\title{
Anabases
}

$\triangle N A B A S E S$ Traditions et réceptions de l'Antiquité

$6 \mid 2007$

Varia

\section{Rome-Babylone. Ruine, corruption, colosse}

\section{Bertrand Prévost}

\section{(2) OpenEdition}

Journals

Édition électronique

URL : http://journals.openedition.org/anabases/3436

DOI : 10.4000/anabases.3436

ISSN : 2256-9421

\section{Éditeur}

E.R.A.S.M.E.

\section{Édition imprimée}

Date de publication : 1 octobre 2007

Pagination : 17-31

ISSN : 1774-4296

\section{Référence électronique}

Bertrand Prévost, "Rome-Babylone. Ruine, corruption, colosse », Anabases [En ligne], 6 | 2007, mis en ligne le 01 janvier 2012, consulté le 20 octobre 2019. URL : http://journals.openedition.org/anabases/ 3436 ; DOI : 10.4000/anabases.3436 
Anabases 6 (2007), p. 17-31.

\section{Rome-Babylone. Ruine, corruption, colosse*}

BERTRAND PRÉVOST

L

L' ART A-T-IL QUELQUE CHOSE À VOIR avec l'idée de corruption ? Les œuvres d'art ontelles à nous apprendre, du moins à nous faire sentir, quant à la corruption ? A priori, pas grand-chose. Et pour une raison bien précise, dont les conséquences sont très importantes pour toute idée à se faire de l'art. C'est que la corruption trouve sa place dans un régime foncièrement naturel ou naturaliste : maladie, pathologie d'un corps vivant non pas causée par un facteur exogène mais en tant que c'est le corps lui-même qui se corrompt. Cela suppose l'action de dynamismes internes, le jeu de puissances, de rapports de force. Or ce régime naturel semble précisément contrevenir au régime passant pour le plus culturel qui soit : le monde de l'art. Plus exactement, c'est à la prétendue autonomie de la fonction symbolique, à son caractère isolé que s'opposerait le régime naturel continuiste.

Pour autant, le discours critique en art - comme discours (donc langage) - s'est souvent plu à mélanger les genres. Certes, le thème ou topos de la "corruption de l'art " n'est pas aussi fréquent que dans le domaine politique, mais le modèle organiciste de l'œuvre d'art ("l'œuvre d'art est comme un corps ", qui remonte au moins à Aristote) aussi bien que le modèle organique de l'histoire de l'art (enfance, maturité, vieillesse un schème historique prégnant dans la critique d'art, au moins depuis Vasari à la Renaissance), donc ces deux modèles ont pu parfois permettre une métaphorique de la corruption. Il est intéressant de ce point de vue que le livre XXV de l'Histoire Naturelle de Pline - celui consacré à la peinture - s'ouvre sur le déclin (osera-t-on dire la corruption ?) de l'art contemporain (Pline l'Ancien écrit dans les décennies centrales du Ier

J'adresse mes plus vifs remerciements à Michel Weemans sans l'aide duquel cette contribution n'aurait jamais vu le jour. 
siècle ap. J.-C.). Pline regrette l'ancien temps des portraits (imagines) supplanté par le luxe débridé, voire débauché de la marqueterie de marbre : "La peinture, art illustre jadis [...] aujourd'hui totalement supplanté par les marbres et en fin de compte également par l'or, au point que non seulement les parois entières en soient couvertes, mais qu'on utilise le marbre découpé et ciselé ou des plaques incrustées dont le dessin contourné représente objets et animaux ${ }^{1}$. " Plus proche de nous, on pensera encore à l'idéologie nazie de l'art dégénéré, qui transposait explicitement l'art sur le terrain de la pathologie : les Demoiselles d'Avignon sont des débiles attardées. Pour ne rien dire, enfin, de ce topos d'un certain discours critique qui depuis presque trente ans consiste à dire : "l'art contemporain, c'est de la merde !", avec Fountain de Marcel Duchamp ou la Merda d'artista de Piero Manzoni en contre-page.

Il est important que ces exemples soient pris dans la critique artistique, autrement dit dans l'élément du langage : c'est dans la bouche de celui qui parle qu'il y a corruption. Cela signifie que l'opposition entre les deux régimes est toujours conservée, et seulement réunie dans l'élément du langage sous forme de métaphore : un simple déplacement. La question - théorique - que je voudrais maintenant poser serait donc celle-ci : la corruption en art doit-elle toujours s'entendre comme une métaphore, un simple déplacement ou bien comme une effectivité, autrement dit, comme une métamorphose?

\section{Argument}

Il faut peut-être chercher la corruption ailleurs que dans l'organique, la dénicher dans le règne apparemment le plus opposé : le minéral, la pierre. Dans l'histoire de l'art, la corruption minérale s'est cristallisée en une figure plastique : il s'agit de la ruine. C'est parce que dans la ruine le monument se délite, se décompose, se désagrège, qu'elle aura pu donner sa figure plastique à la corruption. Il faut tout de suite replacer cela dans l'agencement XVe-XVIe siècles - Rome - Réforme. Dès la fin du XVe siècle, l'anathème lancé contre la corruption de la Rome pontificale par les milieux bientôt réformés ou sensibles aux idées de la Réforme aura pu trouver un riche matériau figural dans le vaste champ de ruines que présentait la Ville. Hypothèse difficile : il ne s'agit pas de dire simplement que la ruine est la conséquence de la corruption, comme si l'une succédait à l'autre. Certes, la Bible fournit plusieurs exemples où la ruine est la conséquence d'un châtiment divin, telle la destruction de Sodome et Gomorrhe. Dans tous ces cas, on ne peut associer véritablement la ruine à la corruption, parce que l'altération (radicale puisqu'il s'agit de la destruction de toute la ville) dépend d'un facteur exogène ou extérieur, et non de l'action d'un processus interne. La ruine ne peut donner à la corrup-

1 Pline l'Ancien, Histoire Naturelle, XXXV, 1, 1, trad. J.M. Croisille, Paris, Les Belles Lettres, 1997, p. 3-5. Les incrustations de marbre coloré sont en réalité bien plus anciennes. 
tion sa plasticité minérale que si ruine et corruption sont en quelque sorte " coprésentes ", autrement dit si la ruine est une manifestation ou une expression de la corruption. En d'autres termes, je voudrais montrer que, pour une certaine sensibilité chrétienne, la corruption morale de l'Église de Rome, était visible dans la plastique de ruine minérale de son siège. Qui plus est, les ruines romaines n'étaient pas n'importe quelles ruines, mais bien celles d'un Empire païen qui portait la corruption dans son cœur, par son idolâtrie, ses mœurs, ses coutumes, son esthétique.

Qu'on se le dise : cette conception de la ruine n'est en rien moderne et humaniste. Elle est même essentiellement réactive. Pourquoi ? Parce que c'est justement à l'humanisme renaissant que l'on doit le tournant décisif qui aura conféré à la ruine une valeur esthétique, et aura permis aux artistes d'en faire un motif artistique. Or, cette invention de la ruine allait de pair avec l'émergence d'une nouvelle conscience historique ${ }^{2}$. La ruine - celle de la Rome antique - n'est pas le fait d'un châtiment divin mais des aléas du temps historique, qui fait et défait les cités. Poggio Braccioloni, par exemple, intitule sa description archéologique des ruines de Rome De varietate fortunae (Les aléas de la fortune) (1448) : « [...] devant l'ancienne grandeur des monuments effondrés et l'immensité des ruines de la cité antique, devant l'ampleur de la chute d'un tel empire, nous admirions du fond de l'âme la stupéfiante et affligeante inconstance de la fortune. [...] Voilà qui est d'autant plus étonnant à décrire et pénible à contempler que la cruauté du sort a bouleversé la forme et la beauté de la Ville au point que, privée de tout ornement, elle gît à terre comme un immense cadavre décomposé et rongé de partout (iaceat instar gigantei cadaveris corrupti) ${ }^{3}$." Cette corruption est désormais conçue comme un processus immanent - une sorte de " temps de la pierre " évoluant au rythme du " temps de la cité " - et non comme une plaie envoyée par Dieu.

Si l'on voit bien le rapport de la ruine à la corruption, on remarque également que se dégage une tension dialectique entre deux types de corruptions pour expliquer la ruine : l'une naturelle, physique et interne ; l'autre surnaturelle, divine et morale. Or je crois que les deux sont profondément liées, et que l'invention des ruines à la Renaissance et le nouveau goût pour la culture archéologique ont pu avoir un effet pervers et rétroactif - sur la conception chrétienne de la ruine. La " renaissance » de l'antiquité païenne par la promotion de ses ruines pouvait, de manière très perverse, alimenter de surcrồt la croyance en une destruction prochaine (c'est une question de puissance) : "Vous, les corrompus, vous serez ruinés; voyez par vous-mêmes le sort qui vous est destiné, vous qui avez bâti le siège de votre Église sur un tas de ruines ».

2 C'est l'argument central du livre de S. ForERO-MENDOZA, Le temps des ruines. Le goût des ruines et les formes de la conscience historique à la Renaissance, Seysell, Champ Vallon, 2002. trad. J.-Y. Boriaud, Paris, Les Belles Lettres, 1999, p. 10. 


\section{Morphologie}

Il y aurait d'abord, ou au moins, un argument en droit pour cette difficile hypothèse, une preuve purement théorique. C'est que la ruine peut donner à la corruption, non pas exactement son esthétique, mais plutôt sa morphologie ou peut-être encore mieux : sa topographie. La corruption politique, me semble-t-il, consiste en des processus de rapports de forces qui prennent la place des rapports de pouvoir ou d'autorité officiels pour reconfigurer un autre agencement socio-politique, officieux celui-là. Mais c'est la même chose avec la corruption que l'on dit morale; on reste dans un régime des influences, des inflexions : l'esprit se laisser affecter par des influences mauvaises, néfastes, qui dévient et infléchissent la droiture de la volonté (l'incorruptible, c'est celui qui ne se laisse pas influencer) ${ }^{4}$. Force ou puissance $v$ s. pouvoir, affect $v$ s. volonté. Tout cela, la ruine le concrétise, en ce sens qu'elle est redistributive, qu'elle opère une nouvelle distribution des places et des éléments. Elle disperse : ce qui était lié devient épars, l'unifié devient disjoint. Elle fait disparaître, mieux elle pulvérise : la pierre retourne à l'état de poussière. Elle inverse ou renverse : ce qui se dressait gît à terre, ce qui était en haut se retrouve en bas. Elle mélange les genres et les règnes : le végétal gagne sur le minéral, l'ouvrage humain (la pierre taillée) en revient à un état naturel (la roche brute). Tous ces dynamismes configurent bien quelque chose, quelque chose qui tient, qui n'est pas chaotique, quelque chose d'individué auquel le nom de "ruine " vient justement donner son unité esthétique. La ruine reste composée : c'est là précisément qu'intervient le travail proprement artistique. Mais ce qui se compose ou s'agence ne le fait plus en fonction des lois tectoniques (la raison du porté-portant) : une architecture sans architectonique, atectonique. De la même manière, la corruption politique dessine comme un vaste souterrain (réseau d'influences) qui ne se décalque pas sur l'architectonique de la société.

Ce que la corruption vient questionner voire critiquer sous la figure de la ruine, c'est en définitive tous les modèles organicistes de la société : elle défait "l'organisme social ", mais n'est pas moribonde pour autant 5 . Mais parler ici d'" organisme ", ou d'" architectonique ", n'est-ce pas encore user de métaphores pour désigner un certain

4 Dans une allégorie morale comme Pallas et le centaure de Sandro Botticelli (Galerie des Offices, Florence), la droiture de Pallas (figure de la Sagesse et de la Vertu) est rendue manifeste par l'aspect parfaitement rectiligne de sa hallebarde, opposé à la sinuosité de l'arc du centaure (figure du vice).

5 Nietzsche a consacré un aphorisme très intéressant de ce point de vue, sur l'étrange vitalité que porte en elle une société corrompue. Cf. F. NieTZSCHE, Le Gai savoir, $₫ 23$, "Les symptômes de la corruption". 
concept de système - qu'il s'agisse d'un corps vivant, d'une société, d'un édifice 6 ? En d'autres termes, la pensée de la corruption n'obligerait-elle pas à une logique non classique des rapports du tout aux parties ? La corruption opère en effet une redistribution des parties en sorte que c'est la nature du tout qui s'en trouve modifiée, sauf que cette affection du tout par la partie se fait en outrepassant «la position respective des parties ».

\section{Iconographie}

L'association de la ruine à l'antiquité païenne corrompue et pécheresse, les images de l'art l'avaient déjà pensée, sous une forme singulière. En effet, les Nativités et les Adorations italiennes, dès le début du Quattrocento, font de la ruine un motif de substitution à une double tradition iconographique qui faisait du lieu de naissance du Christ ou bien une grotte naturelle ou bien un abri rustique. Gentile da Fabriano, dans la célèbre Pala Strozzi (fig. 1), serait sans doute le premier à opérer cette modification. On interprète

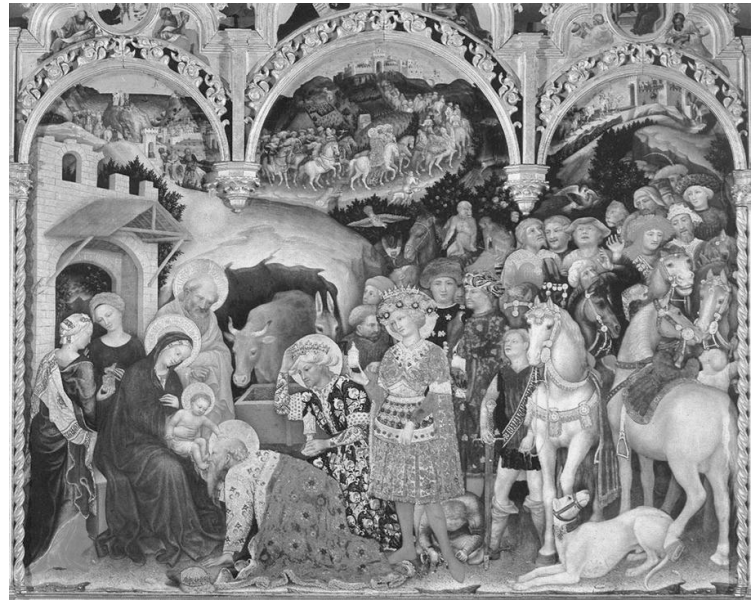

Figure 1. Gentile da Fabriano, Adoration des Mages, 1423, Florence, Galerie des Offices traditionnellement cette différenciation architecturale comme la fin - la ruine donc - de l'Ancienne Alliance au profit d'une nouvelle ère à venir, celle de l'Église, celle de la Nouvelle Alliance. Léglise contre la synagogue. Mais il faut bien faire cas de ce que les Nativités et Adorations italiennes, à partir du milieu du Quattrocento, introduisent presque systématiquement une ruine païenne sous la forme d'un monument antique - à laquelle est adossée

6 C'est sans doute Kant qui est allé le plus loin dans cette interprétation organiciste et architectonique du système. Voir E. KanT, Critique de la Raison pure, "Architectonique de la Raison pure", trad. A. Tremesaygues et B. Pacaud, Paris, PUF, 1993, p. 558 : «Par architectonique j'entends l'art des systèmes. [...] Or j'entends par système l'unité de diverses connaissances sous une idée. Cette idée est le concept rationnel de la forme d'un tout, en tant que c'est en lui que sont déterminées a priori la sphère des éléments divers et la position respective des parties. [...] Le tout est donc un système organique (articulatio) et non un ensemble désordonné (coercervatio) ; il peut, à la vérité, croître par le dedans (per intussusceptionem), mais non par le dehors (per appositionem), semblable au corps de l'animal auquel la croissance n'ajoute aucun membre, mais rend, sans rien changer aux proportions, chacun des membres plus fort et plus approprié à ses fins. " 
une humble cabane (fig. 2). Il faut logiquement y voir non plus une opposition entre les deux Alliances, mais encore une opposition entre le Christianisme appelé à triompher et le paganisme ruiné par lui-même (c'est pour cela que l'on peut légitimement parler de corruption), par ses fausses vérités. Pour autant, l'abri branlant adossé au vestige antique semblera tout aussi ruiné. On ne peut comprendre ce paradoxe apparent que si l'on y voit l'œuvre de l'extraordinaire renversement des valeurs, auquel le christianisme nous a si bien habitués : quelque chose comme une inversion des

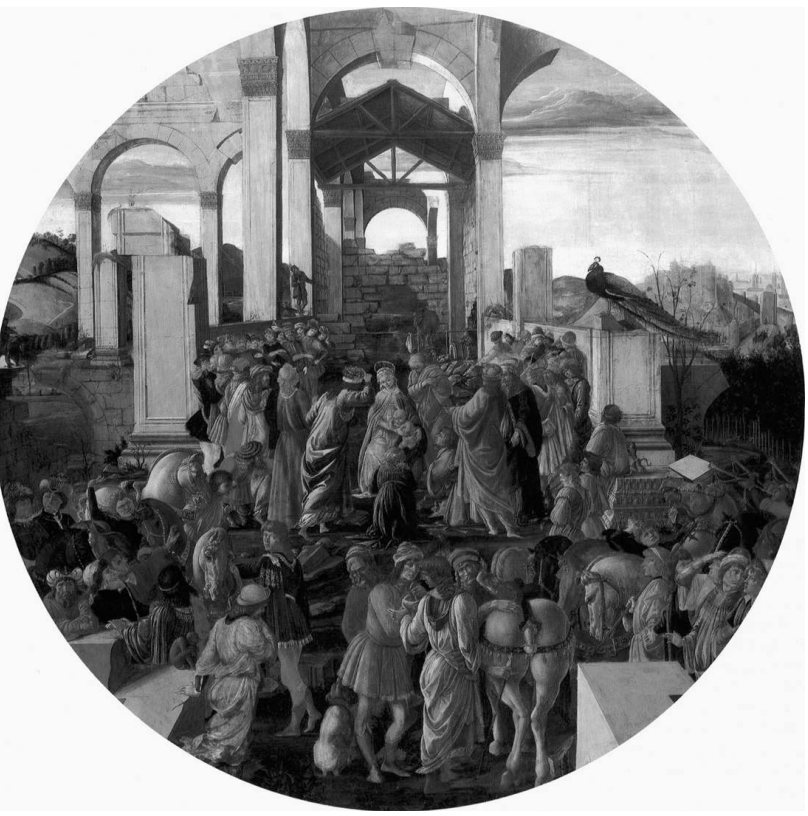

Figure 2. Sandro Botticelli, Adoration des Mages, 1473, Londres, National Gallery valeurs architecturales. Cela donnerait : le noble et arrogant palais de pierre, apparemment fait pour durer (dans le temps) n'est que ruine à l'égard d'une simple cabane, humainement périssable mais divinement éternelle, puisquelle est la matrice de l'Église à venir. L'étable en bois et chaume devient plus solide que le temple de marbre. La

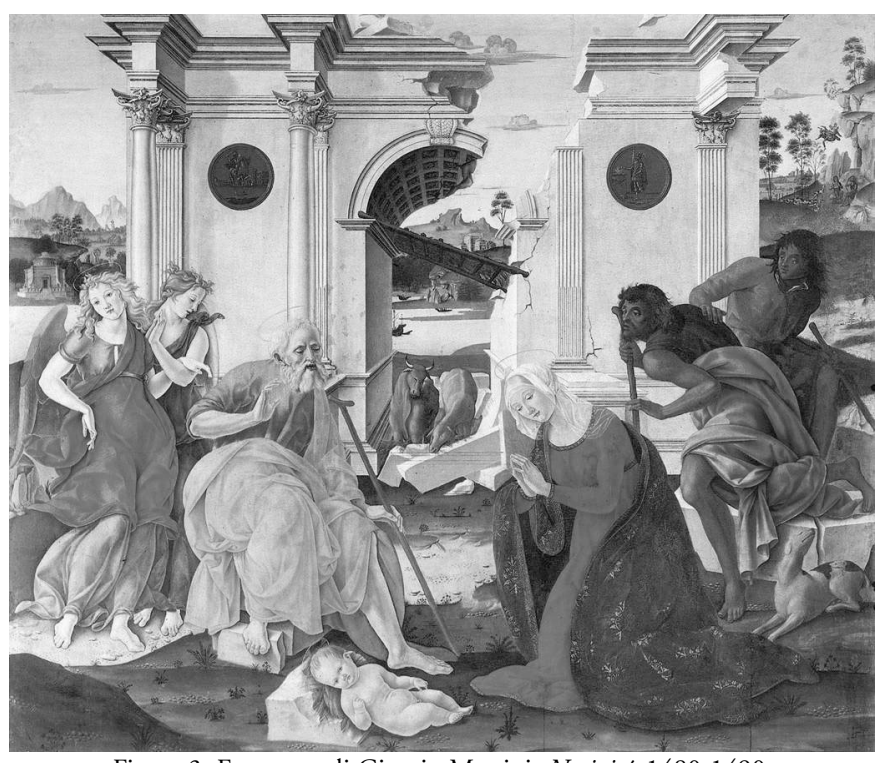

Figure 3. Francesco di Giorgio Martini, Nativité, 1480-1490, Sienne, San Domenico

Nativité de Francesco di Giorgio Martini (fig. 3) le donne puissamment à voir, elle qui introduit un simple abri de bois recouvert de chaume venant se ficher dans la pierre de l'arc de triomphe comme un coin de bois pour faire éclater la roche (voyez l'orientation de la grande cassure dans le monument, qui semble résulter d'un écartèlement dû à l'action de ces quelques poutres). 


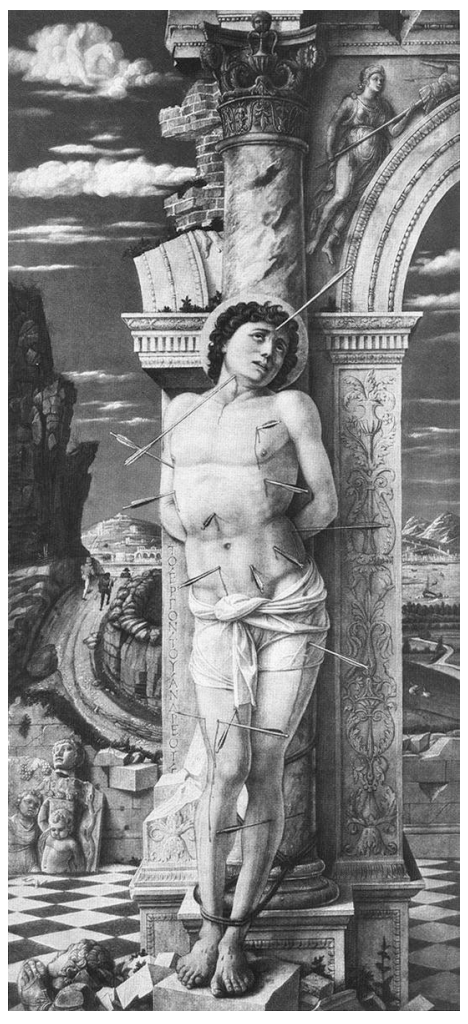

Figure 4. Andrea Mantegna, Saint Sébastien, 1457, Vienne,

Kunsthistorisches Museaum

De ce point de vue, les deux Saint Sébastien de Mantegna, celui de Vienne et du Louvre (fig. 4 et 5), ne font que déplacer cette inversion pour l'introduire dans le monde du corps incarné : le corps de chair du martyr, périssable, corruptible et de fait martyrisé, abîmé, se donne à voir comme l'antithèse d'un autre corps, celui de la statue païenne, dont il a pris l'exacte place : antithèse d'un corps de marbre qui osait prétendre à l'incorruptibilité mais qui gît, morcelé et totalement ruiné.

\section{Poétique}

Revenons à l'hypothèse centrale : la ruine morale de l'Église ne faisait qu'un avec l'état de ruine de son Siège, la Rome antique. Hypothèse autant historique que poétique, parce qu'elle aura été rendue possible par une autre association, très riche et très puissante à sa manière : l'hymen de Rome et de Babylone. RomeBabylone, c'est au début du XVIe siècle, un topos désormais classique de la critique du siège temporel de l'Église ${ }^{7}$. Babylone est très présente dans la Bible, comme ville maudite (lieu d'exil des Hébreux). Dès la Genèse, la construction de l'immense tour est entravée par Dieu en plongeant les bâtisseurs dans la confusion des langues (Genèse, 11, 1-9) ; mais surtout les prophéties de sa destruction pleuvent sur les Livres des Prophètes : "Descends, et assieds-toi dans la poussière, Vierge, fille de Babylone ! Assieds-toi à terre, sans trône, Fille des Chaldéens! On ne t’appellera plus délicate et voluptueuse. [...] Ta nudité sera découverte et ta honte sera vue " (Isä̈e, 47, 1-3) ; " Rangez-vous en bataille contre Babylone tout autour, vous tous, archers - tirez contre elle, n'épargnez pas les flèches " (Jérémie, 50,14); enfin, c'est dans l'Apocalypse que la ruine totale de Babylone s'accomplit définitivement (Apocalypse, 17-19) :

Je te ferai voir la Grande Prostituée, qui est assise sur les grandes eaux [...]. Et la femme que tu as vue, c'est la Grande Ville qui exerce royauté

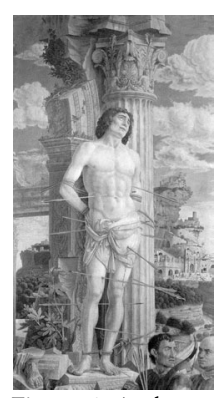

Figure 5. Andrea Mantegna, Saint Sébastien, 1480, Paris, Musée du Louvre

7 Voir A. ChaSTEL, Le sac de Rome, 1527, Paris, Gallimard, 1984, p. 75-120. Voir également O. NICCOLI, Rinascimento anticlericale. Infamia, propaganda e satira in Italia tra Quattro e Cinquecento, Rome-Bari, 2005, p. 22-25. 
sur les rois de la terre ». Suit la chute proprement dite : «Elle est tombée, elle est tombée, Babylone la Grande ! elle est devenue un habitacle de démons, un repaire pour tout esprit impur, pour tout oiseau impur et détesté, parce que du vin de son ardente impudicité elle a abreuvé toutes les nations et que les rois de la terre ont forniqué avec elle et que les marchands de la terre ont tiré richesse du débordement de son luxe. [...] Malheur, malheur à la grande ville ! Babylone, la ville forte ! En un instant est venu ton jugement ! [...] Alors un ange robuste prit une pierre pareille à une grosse meule et la jeta dans la mer en disant : "Ainsi, d'un coup, sera précipitée Babylone la grande Ville”. [...] On entend une voix qui dit «Alleluiah ! [...] parce qu’il a châtié la grande Prostituée qui corrompait la terre par sa prostitution.

Babylone incarne dès lors la Ville terrestre, corruptible et corrompue, par opposition à l'unique et vraie cité de Dieu : la Jérusalem céleste. De fait, il y aurait beaucoup à dire, il me semble, sur le statut chrétien de la ville, plus exactement sur son impossibilité. La seule ville sainte - la Jérusalem Céleste - n’étant justement pas de ce monde. N'oublions pas le fonds pastoral, désertique et nomade de l'Ancien Testament. Le judéo-christianisme n'est pas une religion de la ville mais du désert. À l'inverse, le paganisme - si cette notion a un sens - reste foncièrement attaché à la terre, au sol, à une inscription du sacré dans un lieu défini, délimité. Le dieu païen réside dans son temple, sa ville, son pays (on parlera avec Mircéa Elliade de " point d'hiérophanie fixe »), le dieu judéo-chrétien est partout et nulle part, toujours mobile, transportable - pensons à l'arche de l'Alliance (on parlera alors de "point d'hiérophanie mobile "). Bref, la ville est toujours plus ou moins sujette à méfiance, lieu de tentations, lieu de toutes les corruptions parce qu'implicitement pensée sous un modèle organique, à l'opposé de la minéralité, donc l'incorruptibilité, du désert ${ }^{8}$.

Saint Augustin le premier, sans doute, dans la Cité de Dieu, assimile Rome à une "Babylonia occidentalis 9 ", visant par là la Rome corrompue et décadente des païens. À la fin du XVe iècle, le millénarisme et ses angoisses se joindront à la critique de l'Église romaine pour cristalliser véritablement la figure de Rome-Babylone. Savonarole, par exemple, faisant de Florence une Nouvelle Jérusalem, vouait Rome à la destruction en déclarant dans son sermon du 13 janvier 1495 : "J'ai vu une croix noire dressée sur la Babylone romaine avec l'inscription Ira Domini et il y tombait une pluie de glaives, d'épées et de pierres du ciel [...] tandis qu'une croix d'or descendait des cieux sur Jérusalem ${ }^{10}$. "Et bien évidemment, Luther donnera lui aussi dans le poncif, à commencer par le titre de son traité d'octobre 1520, le Prélude sur la captivité babylonienne de l'église: "Je suis sûr que la domination papale, c'est le règne de Babylone et

8 Voir notamment les belles analyses de R. Debray, Dieu, un intinéraire, Paris, Odile Jacob, 2001, p. 119-149.

10 Cité par A. CHASTEL, Art et humanisme à Florence au temps de Laurent le Magnifique, Paris, PUF, 1982, p. 396. 


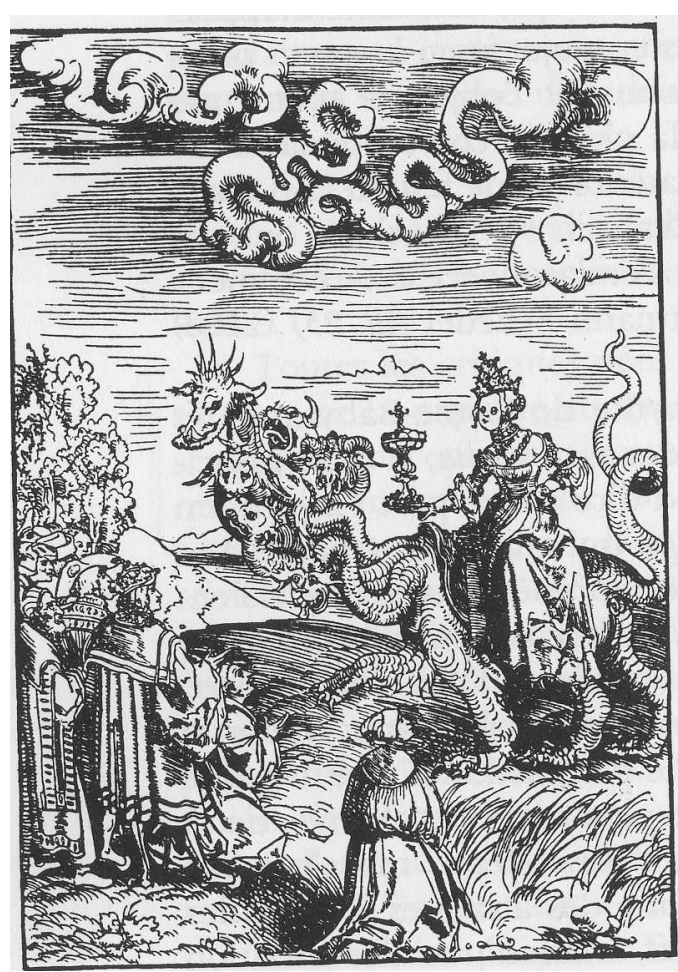

Figure 6. Lucas Cranach, La Grande Prostituée de Babylone, in September Testament, Wittenberg, 1522, Paris, Bibliothèque nationale du roi Nimrod, puissant chasseur." Qui plus est, le grand chantier romain du moment entrepris par Jules II, à savoir la reconstruction de la basilique Saint Pierre, ouvrage colossal, entreprise démesurée, ne pouvait apparaître aux yeux des milieux en passe d'être réformés que comme une nouvelle tour de Babel. André Chastel a très justement parlé d'une sorte de "sacralisation inversée » pour penser toute la puissance, toute la charge d'une « désacralisation de Rome <qui> ne pouvait s'opérer qu'à travers ce qu'on pourrait appeler une "diabolisation", c'est-àdire une sacralisation inversée. Toute la chrétienté gravitait autour de cette ville, qui était le but de tant de pèlerinages, grâce auxquels l'imago Urbis s'était répandue dans l'Occident ${ }^{11}$ ".

Les images sont pleinement partie-prenante de toutes ces dénonciations et accusations au nom de l'association diabolique Rome-Babylone.

On savait l'importance de l'imprimerie dans l'essor de la Réforme, et de manière équivalente, la gravure a joué un rôle non négligeable non pas tant dans la diffusion de sa doctrine que dans la publicité de son positionnement stratégique vis-à-vis de Rome : une véritable guerre dans les images (peut-être d'ailleurs la première forme de véritable propagande qu'ait connue l'Occident). Une image assez commune de cette propagande anti-papiste consistait à figurer la Grande prostituée de Babylone, dans l'Apocalypse, coiffée de la tiare pontificale : allusion directe à la corruption du Saint-Père lui-même. Cette image se trouve par exemple dans la Bible de Luther de 1522, le September Testament illustré par Cranach (fig. 6), ou encore dans une autre gravure, due à Hans Holbein, exécutée pour la Bible de Thomas Wolff (Bâle, 1523) (fig. 7).

Mais l'association de la chute de Babylone avec la destruction de Rome est rendue explicite dans l'une des illustrations de cette même Bible allemande de Luther de 1522. Seule l'Apocalypse est illustrée de gravures dues à Lucas Cranach et à son atelier (fig. 8). Il s'agit plus particulièrement de la planche XIV qui illustre la destruction de Babylone. La ville qui voit ses monuments détruits, ou plutôt renversés, n'est autre que Rome elle- 


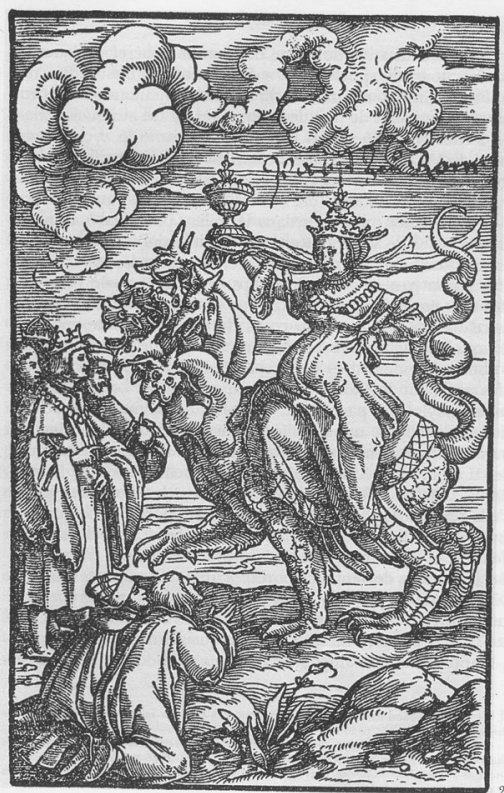

Figure 7. Hans Holberin, Grande prostituée de Babylone, in Bible de Thomas Wolff, 1523, Paris, Bibliothèque nationale

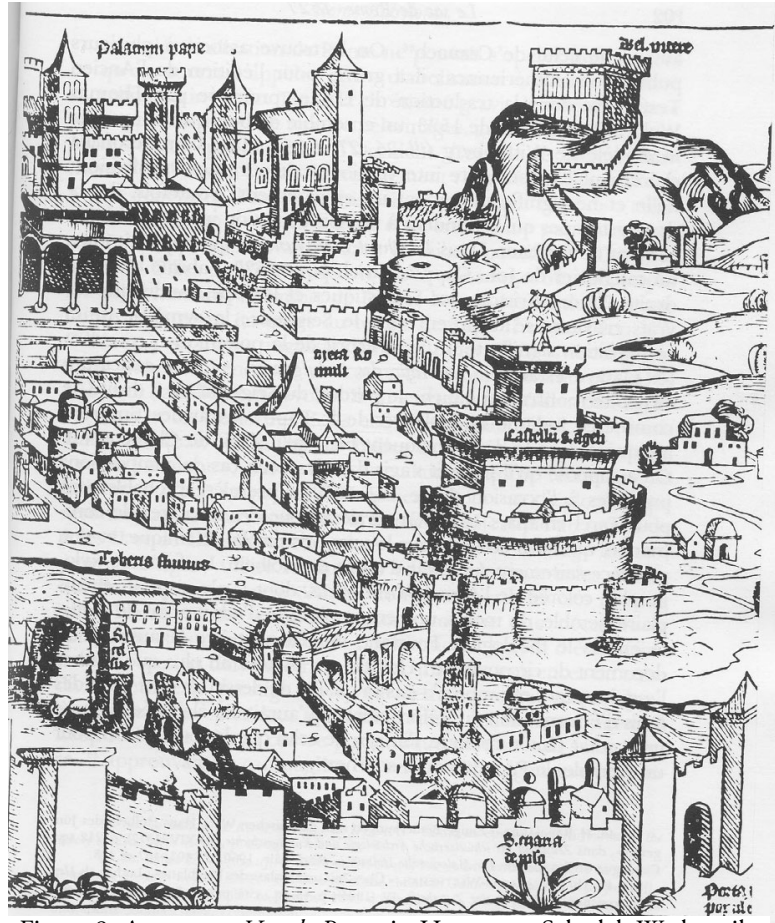

Figure 9. Anonyme, Vue de Rome, in Hartmann Schedel, Wetkronik, Nuremberg, 1493

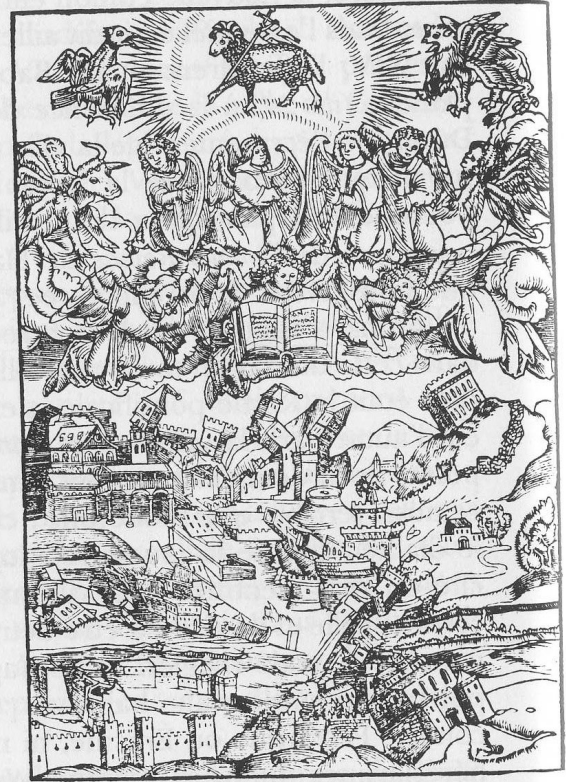

Figure 8. Lucas Cranach, La destruction de Babylone, in September Testament, Paris, Bibliothèque nationale

même. Cranach s'est tout simplement contenté de récupérer une ancienne vue de Rome une gravure anonyme visible dans la Weltkronik d'Hartmann Schedel (Nuremberg, 1493) dont il a ruiné les principaux édifices (fig. 9). Ce n'est donc pas seulement l'institution pontificale qui est la cible des critiques, mais bien la ville de Rome, dans ses pierres, ses murs, ses monuments.

\section{Peinture}

Cela étant, il n'est pas encore question de ruine à proprement parler, puisque ce que les trompettes des anges renversent, ce sont encore des monuments 


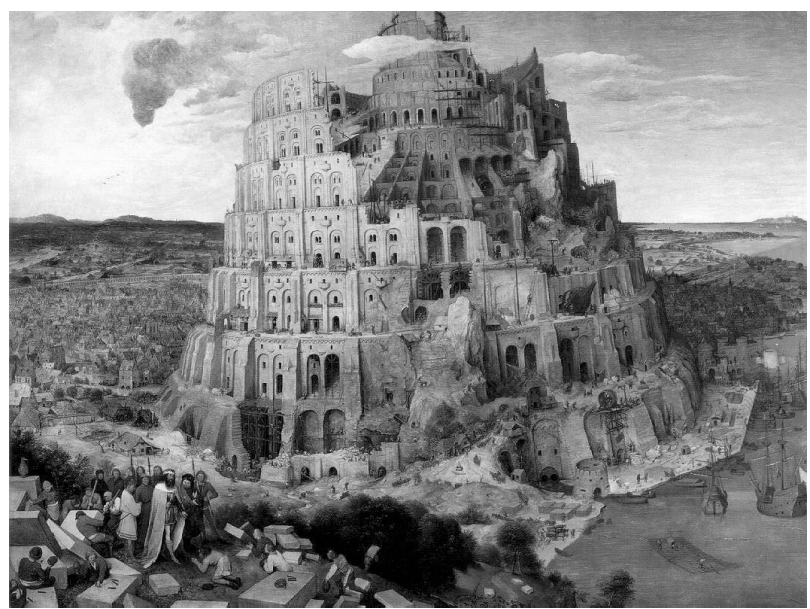

Figure 10. Bruegel l'Ancien, La Tour de Babel, 1563, Vienne, Knsthistorisches Museaum

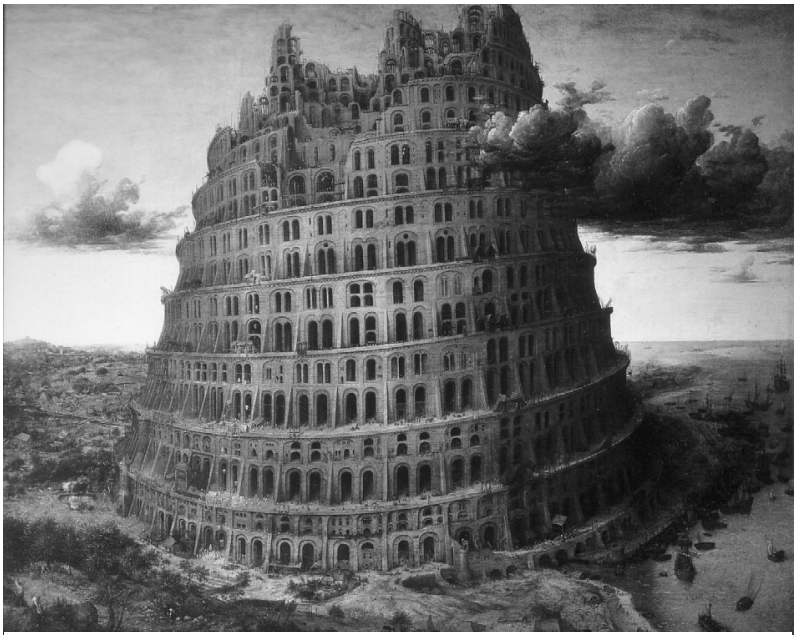

Figure 11. Bruegel l'Ancien, La petite Tour de Babel, 1563 (ca.), Rotterdam, Museum Boijmans Van Beuningen plus ou moins modernes, qui ne sont certes pas en ruine dans les premières décennies du XVI e siècle. L'image qui permet d'articuler pleinement Rome-Babylone à la ruine, la voici : c'est la Tour de Babella grande - de Bruegel (fig. 10). Le peintre anversois l'a prise pour thème dans deux tableaux peints autour de 1563, l'un conservé à Vienne, l'autre (fig. 11) - sans doute légèrement postérieur - de dimensions plus réduites conservé à Rotterdam (d'où son titre courant de Petite Tour). Au premier plan, le roi de Babylone Nemrod entouré de ses suivants effectue une visite sur le chantier démesuré qu'il a entrepris : l'édification d'une tour si haute qu'elle crève les nuages. De fait, Bruegel insiste sur le gigantisme du bâtiment en l'inscrivant dans un paysage panoramique qui s'accorde, par son immensité, à sa taille (voir notamment l'ombre portée à droite). Partout s'affairent des ouvriers, des maçons, des tailleurs de pierre, partout des échafaudages, roues, échelles, appareils d'élévation... La précision du détail est impressionnante. La Petite tour, quant à elle, outre le fait qu'elle présente un monument beaucoup plus achevé, a abandonné la scène du premier plan. Ces deux tableaux sont le point de départ d'une longue tradition iconographique ${ }^{12}$.

12 Pour un catalogue iconographique de la Tour de Babel, voir H. MinKOwsKI, Turm zu Babel, s.l., Luca Verlag Freren, 1991. On pourra également se rapporter au dossier "L'ombre de la Tour", FMR, avril-mai, 2004, p. 29-50. 
Il va de soi, comme il arrive très souvent dans la peinture flamande et dans celle de Bruegel l'Ancien en particulier, que le propos est ici moral : la peinture se veut jugement. Si l'on raisonne en termes purement iconographiques (i.e. dans l'élément de la représentation), ce jugement est porté par le texte biblique : vanité d'une construction humaine voulant rivaliser avec la création et la grandeur divines, signe d'une cité corrompue qui ne tardera pas à être

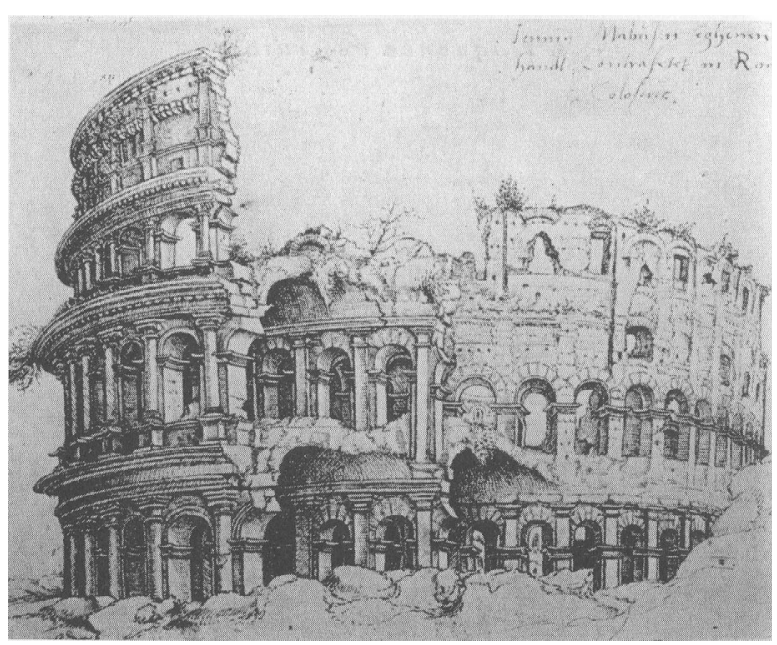

Figure 12. Mabuse, Le Colisée, 1509 détruite - je ne reviens pas sur la lettre biblique. Mais comment, figuralement (et non plus iconographiquement) la peinture accomplit-elle ce jugement moral ? Comment la peinture de Bruegel produitelle (et non plus représente-t-elle) l'union de Rome et de Babylone ? Il s'agit d'une invention très singulière. C'est en effet dans la forme même du monument que vient se nicher quelque chose de Rome, quelque chose de sa corruption, quelque chose de païen, quelque chose de ruiné - j'entends : le Colisée (fig. 12). Le peintre anversois s'est en effet manifestement inspiré de la forme du célèbre monument romain, cela a toujours été remarqué. On y retrouve "les travées de doubles arcades, s'enroulant en spirale autour d'architectures imbriquées, rappel(ant) les rangées d'arcs superposés, les dégagements et vomitoires du grand cirque romain 13 ». Bruegel avait de ses yeux vu le monument puisqu'il avait effectué un voyage en Italie en 1552, et avait séjourné à Rome en 1562 (le tableau, de 1563 est donc intimement lié à l'expérience du voyage romain). Formellement, le tableau en finit avec l'ancien schème qui faisait de la Tour de Babel un édifice quadrangulaire ${ }^{14}$.

Point n'est besoin de s'attarder sur le lien presque consubstantiel entre Rome et le Colisée. Le monument n'a pas eu à attendre les investigations archéologiques du Quattrocento pour réapparâtre... : il n'avait pas cessé d'être là. La nouveauté, avec l'humanisme renaissant, c'est que l'on déplore son état de délabrement. Poggio Bracciolini peut écrire, dans son De varietate fortunae déjà cité : «La ville regorgeait de théâtres et d'amphithéâtres destinés aux jeux donnés pour le peuple. Immense et plus beau que tous

13 S. Forero-MendozA, Le temps des ruines [n. 2], p. 124.

14 Sur le rapport entre le tableau de Bruegel et le Colisée, voir i.a. Z. WAZBINSKI, "La construction de la Tour de Babel par Bruegel le Vieux. Genèse d'un symbole d'urbanisme”, Bulletin du Musée National de Varsovie, V, 1964, n 3-4, p. 112-121. 
les autres était, dit-on, l'ouvrage en pierres de Tibur, élevé pratiquement au cœur de Rome par le divin Vespasien, et communément appelé Colisée : vu la sottise des Romains, on l'a en grande partie détruit pour faire de la chaux ${ }^{15}$. " Le Colisée s'imposait comme la ruine paradigmatique de la Rome antique. Quand Marten van Heemskerck - l'un des "Flamands à Rome » (ou Romanistes) les plus importants - fait son autoportrait en 1553, presque en guise de testament artistique, c'est tout logiquement devant le Colisée qu'il pose (fig. 13), « ou plus exactement devant un vieux tableau qu'il avait fait du Colisée, en se remémorant sa jeunesse, du temps où il dessinait en ce lieu 16 ».

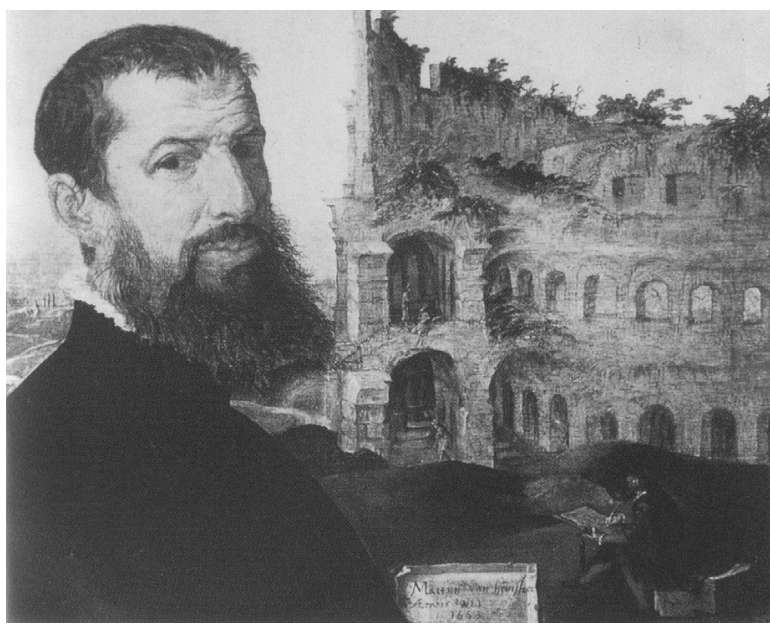

Figure 13. Marten van Heemskerck, Autoportrait devant le Colisé, 1553,Cambridge, Fitzwilliam Museum

Mais la gloire archéologique et artistique du monument ne doit pas masquer la pluralité des points de vue - et notamment du point de vue chrétien. Il faut bien penser la position très marginale de Bruegel au regard de l'enthousiasme flamand du milieu du XVI ${ }^{\mathrm{e}}$ siècle pour le paysage de ruines romaines. Certes, les enjeux sont avant tout esthétiques, Bruegel cherchant à inventer un nouveau type de paysage, mais n'oublions pas non plus tout simplement - sa ferveur de sujet chrétien. En un mot, n'oublions pas que le Colisée représentait aussi le lieu par excellence de la corruption des mœurs païennes : le lieu des jeux du cirque. Aucun lettré du XVI iècle ne pouvait oublier les anathèmes proférés par Tertullien (De spectaculis) ou saint Augustin : ironiquement (permettant implicitement une double condamnation) "Sachez donc, vous qui l'ignorez, et vous aussi qui feignez l'ignorance, n'oubliez pas, au milieu de vos murmures contre votre libérateur, que ces jeux scéniques, spectacles de turpitude, œuvres de licence et de vanité, ont été établis à Rome, non par la corruption des hommes, mais par le commandement de vos dieux ${ }^{17}$. "

Mais surtout, c'est la nature ruinée du Colisée, avant sa nature monumentale qui permettait à Bruegel de proposer une pensée rien que picturale de la corruption. La géniale invention rendait possible l'articulation de deux séries causales et antagonistes de ruine :

15 Le POGGe, Les ruines de Rome, I, 5 [n. 3], p. 32.

16 N. Dacos, Roma quanta fuit ou l'invention du paysage de ruines, Bruxelles, Paris, Musée de la Maison d'Érasme, Somogy, 2004, p. 85.

17 Saint Augustin, La cité de Dieu, I, 32. 
- l'une naturelle et interne : l'édifice tombe de lui-même sous l'effet du temps. C'est la ruine telle que l'humanisme archéologique la pense : la ruine comme corruption du temps, immixtion de la Fortune dans les choses mêmes. La ruine comme signe d'une corruption physique ;

- l'autre surnaturelle et externe : la destruction est ordonnée par Dieu, la ruine est la conséquence d'un châtiment divin. La ruine est cette fois le signe d'une corruption morale.

Cette distinction se rejoue poétiquement dans le couple Colisée-Tour de Babel/Babylone, le premier ruiné par la puissance corruptrice du temps, la seconde ruinée par une intervention divine (confusion des langues pour la Tour, anéantissement total pour la ville).

Cette rencontre entre les deux séries causales de la ruine est véritablement assurée par le colosse, par la forme colossale, voilà l'idée géniale de Bruegel. Le point commun formel du Colisée et de la Tour de Babel, c'est bien leurs dimensions colossales. On sait que l'étymologie, pour l'amphithéâtre de Flavien, est trompeuse et que son nom dérive (tardivement, au Moyen Âge) de la présence juste à côté d'une statue colossale en bronze de Néron. Mais précisément, tout cela fait système. Parce que le colosse est aussi une question de peinture, au moins depuis le De pictura de Leon Battista Alberti. Tout se passe en effet comme si Bruegel avait pris à la lettre l'injonction du peintre-humaniste, sans trop savoir si c'était pour s'en accommoder ou le réprouver. Au paragraphe 35 de son traité, dans un très important développement sur la composition, le peintre humaniste enjoignait en effet que « le grand œuvre du peintre n'est pas le colosse, mais la représentation d'une histoire (historia). En effet, la représentation plus qu'un colosse porte à l'éloge du talent (ingenium) 18 ". Autant le propre de l' historia est d'être composée, c'est-à-dire de synthétiser des parties dans un tout univoque (l'historia), autant le colosse est monolithique, non composé, sans parties, ou plutôt - c'est là que cela devient intéressant pour nous - n'a de parties qu'à l'état de ruine : ses parties n'existent que comme fragments. Dit en d'autres termes, le colosse est destiné à la ruine, et s'il est destiné intensivement à la ruine, c'est parce qu'il est déterminé par une double corruption. Il est d'abord signe de corruption morale, démesure humaine et vanité, et s'expose ainsi au châtiment surnaturel. Mais encore, par ses dimensions colossales, il perd toute mesure humaine, je veux dire qu'il s'ouvre, qu'il s'expose (comme une puissance mathématique) à une dimension naturelle, voire cosmique - au risque d'être affecté par des puissances naturelles ou élémentaires, au risque d'être physiquement corrompu.

Alberti comme Bruegel n'étaient certainement pas sans ignorer ce passage du livre XXXV de l'Histoire naturelle de Pline où il est question d'une peinture colossale de Néron : "Je ne passerai pas non plus sous silence cette folie de notre époque dans le domaine pictural. L'empereur Néron s'était fait peindre en des proportions colossales 2004, p. 128-129. 
sur un toile de lin de 120 pieds : acte inouï jusqu'alors. Cette peinture, une fois achevée dans les jardins de Maius, fut frappée par la foudre et brûla avec la plus belle partie de ces jardins ${ }^{19}$. " Par sa démesure, le portrait impérial perd la protection " isolante " de son format de tableau pour s'exposer aux aléas cosmiques (météorologiques) et être détruit par la foudre. On est passé d'un régime à un autre : du domaine clos, symbolique et moral de l'art au domaine naturel, ouvert et extériorisé des puissances cosmiques et élémentaires (eau, terre, feu, pierre). Mais il est évident que la foudre, dans le passage de Pline, peut aussi être interprétée comme châtiment jupitérien Néron faisant la figure idéale du tyran corrompu, ce qui ramènerait une fois encore le colosse au point d'articulation entre la corruption physique et la corruption morale.

Comprenons bien que pour Bruegel, ces problèmes étaient de nature artistique, intimement liés à la promotion d'un type de " paysage cosmique " : vastes visions panoramiques s'étendant presque à l'infini. Tout concourt donc, dans La Tour de Babel, à " paysager " ou à " paysagifier " le monument, c'est-à-dire à lui donner des coordonnées naturelles, à l'inscrire dans un régime naturel ouvert à la corruptibilité des influences terrestres, célestes, océaniques... Elle touche le ciel et se perd dans les nuages; elle domine la terre et la mer en les ombrageant sur une très vaste étendue (voir l'ombre portée à droite) ; de la montagne elle a la hauteur (elle s'élève au-dessus de l'horizon comme les montagnes au très lointain) mais aussi la minéralité : elle est bâtie sur un roc, littéralement sculptée dans la roche, laissée encore brute en maints endroits ${ }^{20}$. Ce dernier trait est évidemment capital car il destine explicitement la tour à sa ruine, en signalant l'imminence du retour de la pierre taillée (et la scène du premier plan est là pour $\mathrm{y}$ insister) à la roche informe. Bref, la tour fait corps avec son paysage, elle flue avec ses coordonnées élémentaires. Tout se passe comme si le style de Bruegel dans ce genre particulier de paysage cosmique venait donner le change artistique ou esthétique aux différentes puissances convoquées par les discours : le souffle de l'Histoire (grandeur et décadence de Rome) ; la superbe d'édifices monumentaux ; la portée cosmique des imprécations, malédictions et autres exécrations prophétiques, qui convoquent toujours la terre entière ; le vibrant écho des trompettes de l'Apocalypse sonnant le commencement de la vengeance divine... Les grandes dénonciations de la corruption ont sans doute quelque chose de cela même qu'elles mettent à l'index : leur réceptivité à l'influence des puissances - c'est cela qui les rend si fascinantes.

\begin{tabular}{l} 
Bertrand PRÉVOST \\
\hline ATER à l'Université de Provence \\
136, rue Ordener \\
75018 Paris \\
bertrand.prevost@wanadoo.fr
\end{tabular}

